\title{
ANÁLISIS
}

\section{CONOCIMIENTO EN LA NUBE: EVOLUCIÓN DE LAS} INTRANETS

\author{
Pablo Lara-Navarra y David Maniega-Legarda
}
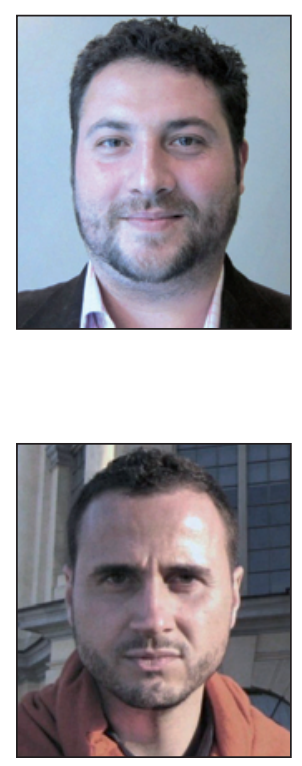

Pablo Lara-Navarra, licenciado en documentación por la Universidad de Granada y doctor por la Universidad Pompeu Fabra, realizó el Master de Sociedad de la Información y Conocimiento de la Universidad Oberta de Catalunya (UOC). Su carrera se centra en la docencia, innovación en productos y consultoría a empresas. Es especialista en gestión de innovación, gestión de conocimiento y elearning, temas sobre los que ha publicado numerosos libros y artículos. Ha dado conferencias en más de 10 países y es asesor de empresas tecnológicas. Ha sido director académico del Master de Tecnologías Accesibles UOC-Technosite. Es director de Innovación de la UOC y profesor de los Estudios de Ciencias de la Información y de la Comunicación de dicha universidad a distancia.

Universitat Oberta de Catalunya plara@uoc.edu

David Maniega-Legarda, historiador del arte y documentalista técnico, es director de la Oficina Abierta de Innovación de la Universitat Oberta de Catalunya (UOC). Ha sido técnico webmaster en la UOC durante diez años y responsable del Equipo web del Servicio de Cooperación Bibliotecaria del Depto. de Cultura y Medios de Comunicación de la Generalitat de Catalunya. Actúa como consultor de usabilidad y accesibilidad web, estrategia tecnológica y procesos de innovación en las organizaciones. Es experto en la creación y desarrollo de proyectos de innovación centrados en e-learning. Es profesor en el Máster Calsi de la Universidad Politécnica de Valencia y en el Máster de documentación digital de la Universitat Pompeu Fabra donde además es coordinador de proyectos web.

Universitat Oberta de Catalunya dmaniega@uoc.edu

\section{Resumen}

Los sistemas de información actuales evolucionan con tanta rapidez que incluso aparecen y desaparecen sin que se lleguen a percibir sus efectos, pues emergen sistemas nuevos sin que se hayan podido desarrollar completamente los anteriores. Este trabajo realiza un estudio del concepto de intranet en el área de información y documentación que permite observar su evolución, métodos y principales características técnicas. Se establece cómo desde el principio las intranets están vinculadas con la tecnología web, y cómo en la actualidad el cloud computing interviene en las nuevas formas de entenderlas. Por último se muestran dos ejemplos de intranet en cloud computing híbrido y privado.

\section{Palabras clave}

Intranet, Cloud computing, Tecnología en la nube, Evolución de las intranets, Gestión de información en la nube.

\section{Title: Knowledge in the cloud, evolution of intranets}

\begin{abstract}
Current information systems evolve very rapidly, even appearing and disappearing imperceptibly, as new systems emerge without having been able to fully develop the previous ones. This paper provides a study of the concept of intranet in the area of information and documentation, describing its conceptual evolution, methods and main technical characteristics. We establish how from the beginning intranets have been based on web technology, and how today cloud computing allows new ways to understand them. Finally, two examples of intranet in hybrid and private cloud computing are shown.
\end{abstract}

\section{Keywords}

Intranet, Cloud computing, Technology in the cloud, Intranet development, Information management in the cloud.

Lara-Navarra, Pablo; Maniega-Legarda, David. “Conocimiento en la nube: evolución de las intranets”. El profesional de la información, 2011, marzo-abril, v. 20, n. 2, pp. 175-181.

DOI: 10.3145/epi.2011.mar.07

Artículo recibido el 04-01-11

Aceptación definitiva: 23-02-11 


\section{Introducción}

Las tecnologías de la información en torno a internet evolucionan con gran rapidez, incluso aparecen y desaparecen sin que se hayan podido explotar sus posibilidades, pues en seguida emergen otras nuevas. Las intranets no están exentas a esta dinámica y durante esta primera década del siglo XXI ha surgido un conjunto de técnicas que inciden directamente en su diseño.

La fuente de conocimiento cooperativo Wikipedia (2011) describe una intranet como "una red de ordenadores privados que utiliza tecnología internet para compartir dentro de una organización parte de sus sistemas de información y sistemas operacionales". Las intranets, pues, evolucionan al ritmo del avance de las TICs (Pérez; Solana, 2006).

El presente trabajo hace un pequeño recorrido histórico de las intranets, tanto a nivel conceptual como metodológico y tecnológico, y ofrece una nueva visión con la incorporación del cloud computing.

\section{Visión retrospectiva de las intranets documentales}

Nos remontaremos 12 años atrás, en 1998, cuando la extinguida Socadi ${ }^{1}$ organizó una reunión que fue documentada por la revista EPI (Abadal, 1998). Un grupo de expertos opinaron sobre un concepto entonces novedoso: "intranet", y dibujaron un estado del arte exponiendo qué se entendía por intranet documental en ese momento tecnológico y temporal. Lluís Codina indicaba como característica más destacable de la intranet "su gran capacidad de integración", y referenciaba como sus bondades las siguientes: "desde un sólo programa (el navegador) se pueden consultar informaciones muy distintas (documentos de todas clases, bases de datos, etc.); se eliminan las diferencias entre sistemas locales y remotos; y aparecen nuevas posibilidades de relación entre los sistemas de recuperación de la información y los hipertextuales, así como entre información y comunicación".

Por otra parte Josep-Àngel Borràs definió que la característica básica de la intranet era su "modelo de arquitectura de red que usa protocolos y estándares abiertos, transparente con internet y que dispone de un cliente universal (el navegador)". Adentrándose y perfilando algunos servicios, argumentó que la intranet posibilitaba "gestionar todos los documentos que una organización va produciendo (textos, correo electrónico, hojas de cálculo, etc.) y también todas sus aplicaciones (bases de datos, etc.)".

Por último destacar los comentarios del propio Abadal, que resaltaba que una las ventajas de poner en marcha una intranet estaba en el coste, pues se podía iniciar con pocos medios, e incluso a pequeña escala como prueba piloto. $A$ su vez profundizó en la estructura organizativa y sostuvo que las intranets por su carácter democratizador encajaban sobre todo en instituciones con organigramas en red y/o pirámides de mando planas.

En el año 2002 bajo el título "Intranets: sonrisas y lágrimas", Adela d'Alòs-Moner publicaba un artículo donde exponía que los sistemas de información desarrollados bajo mode- los de intranet estaban en plena explosión, resultado de factores como:

- Aprovechar la infraestructura existente de redes y ordenadores.

- Bajo coste de implantación.

- Basarse en una administración centralizada pero conectando a todos con todos.

- Rapidez de su puesta en funcionamiento.

Por último queremos citar el artículo "Definición del modelo estratégico de desarrollo de una intranet corporativa" (Bustelo-Ruesta; García-Morales; García-de-Paso, 2006), donde se expone por primera vez la existencia de ciclos: una primera etapa donde la intranet destaca por ser una herramienta orientadora y facilitadora de información para empleados, como un sistema para la mejora de comunicación vertical de la organización y, de ayuda para la publicación de información y documentación corporativa. En una segunda etapa las intranets se centran en mejorar la eficacia y eficiencia del conocimiento interno, se introducen elementos para aumentar la bidireccionalidad en la comunicación, entre personas y unidades organizativas $y$, aparecen las primeras herramientas para descentralizar la intranet. Y el último ciclo es una etapa centrada en la reflexión y rediseño desde una perspectiva estratégica de gestión global e integrada de la información de la organización.

Hecha esta corta revisión histórica, nos planteamos dos cuestiones sobre el uso de las intranets:

1. ¿Cómo se puede hacer pública la información trabajada de forma interna?, ¿qué sistema permite que ello sea fácil sin romper el concepto intranet?

Para resolver esto vamos a plantear una solución híbrida, presentando un ejemplo basado en SharePoint.

2. Además de ser un espacio privado, la intranet se basa en protocolos de internet. ¿Qué sucede cuando evolucionan?, ¿cuáles son las nuevas tecnologías que pueden incidir en las intranets?

Para este planteamiento veremos elementos comunes entre intranets y cloud computing, presentando un concepto "nuevo" de intranet basado en la llamada "nube privada".

SharePoint, de Microsoft, es uno de los programas más usados de creación y gestión de intranets

\section{SharePoint, una solución híbrida para intranets complejas}

Uno de los programas de creación y gestión de intranets corporativas más usado en organizaciones es SharePoint, tal y como se desprende en los últimos informes de Nielsen "10 best intranets" de los años 2009, 2010 y 2011-. A nivel interno ofrece buenas garantías de seguridad, conformidad, privacidad y compatibilidad de aplicaciones, y desde su última versión las ofrece también en su modelo para trabajar nube (en servidores remotos, como se verá más adelante). 
Es una plataforma de colaboración organizacional, un gestor de contenido empresarial, capaz de integrarse con las diferentes herramientas de Microsoft (software servidor, cliente de correo, suite ofimática, etc.), confiriéndole un gran potencial para gestionar una intranet, especialmente en aquellas organizaciones en las que ya se utilizan productos de Microsoft.

Con SharePoint se puede:

- implementar aplicaciones a medida;

- configurar los privilegios para el acceso a la información necesaria e imprescindible para cada objetivo y proceso;

- crear y gestionar una extranet;

- tener un gestor documental;

- tener un sistema con políticas de pu-

blicación (flujos de trabajo, plantillas de edición, colaboración grupal, escenarios de portales, etc.).

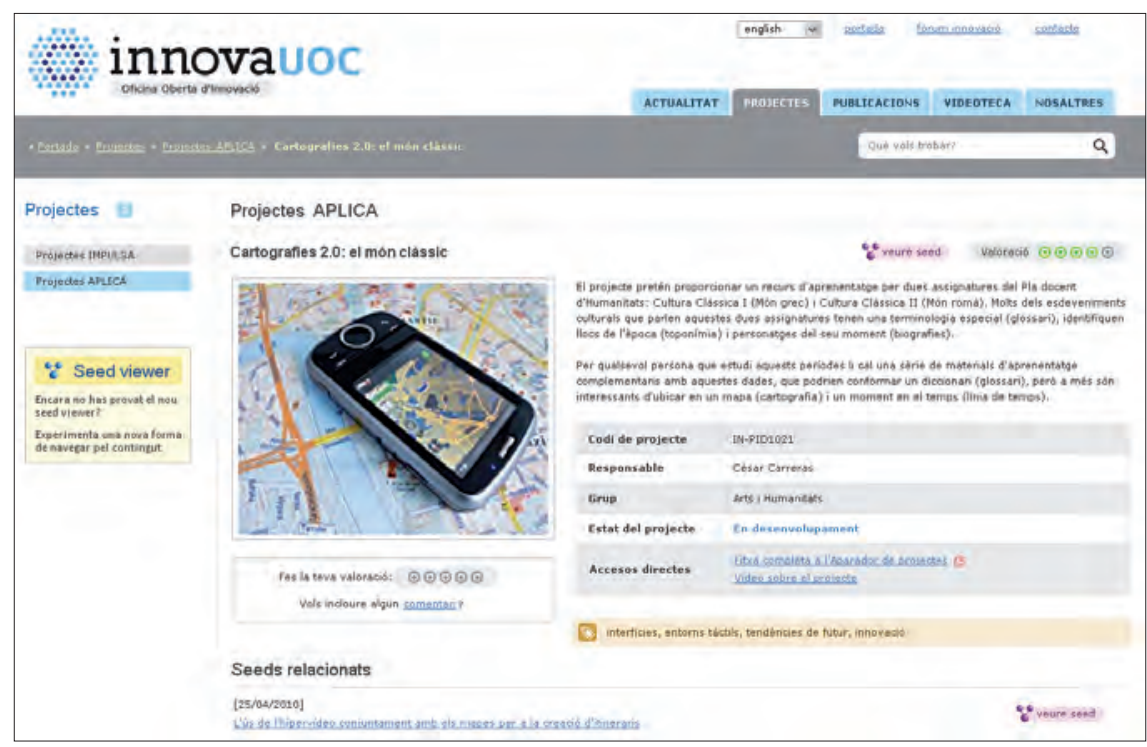

Figura 1. Ficha de proyecto vista desde la intranet

De todas las características de SharePoint queremos destacar la que da respuesta a la primera cuestión planteada antes -publicar información generada internamente-, tomando como ejemplo el caso de la Oficina Abierta de Innovación de la Universitat Oberta de Catalunya (UOC). Para ello nos focalizamos en dos elementos clave, como son la administración del contenido para la generación de portales y los mecanismos de colaboración.

\section{Administración de contenido para crear un portal web}

Una de las ventajas de una intranet es poder controlar los flujos de la documentación, determinar políticas de acceso, modificar, eliminar y publicar documentos, etc. Muchos de esos documentos pueden publicarse en la web, ya sea en un sitio aislado o en un portal vertical. En ese sentido SharePoint 2010 es útil para diseñar, distribuir y administrar contenidos mediante modelos de publicación automática en portales de la intranet, sitios web de presencia corporativa en internet, y portales departamentales aislados sin dependencia jerárquica con el resto de la estructura de la intranet. Gracias a esto se elimina la posibilidad de duplicidades cuando nos enfrentamos a la publicación de contenidos fuera de nuestra intranet.

Al trabajar de forma totalmente integrada se pueden establecer procesos paralelos pero con fines diferentes bajo la misma estructura, definiendo qué "islas" documentales son las que van a tener una visibilidad pública fuera de la intranet. La capacidad para crear y publicar contenido web de forma automática, basándonos en plantillas predefinidas según las necesidades

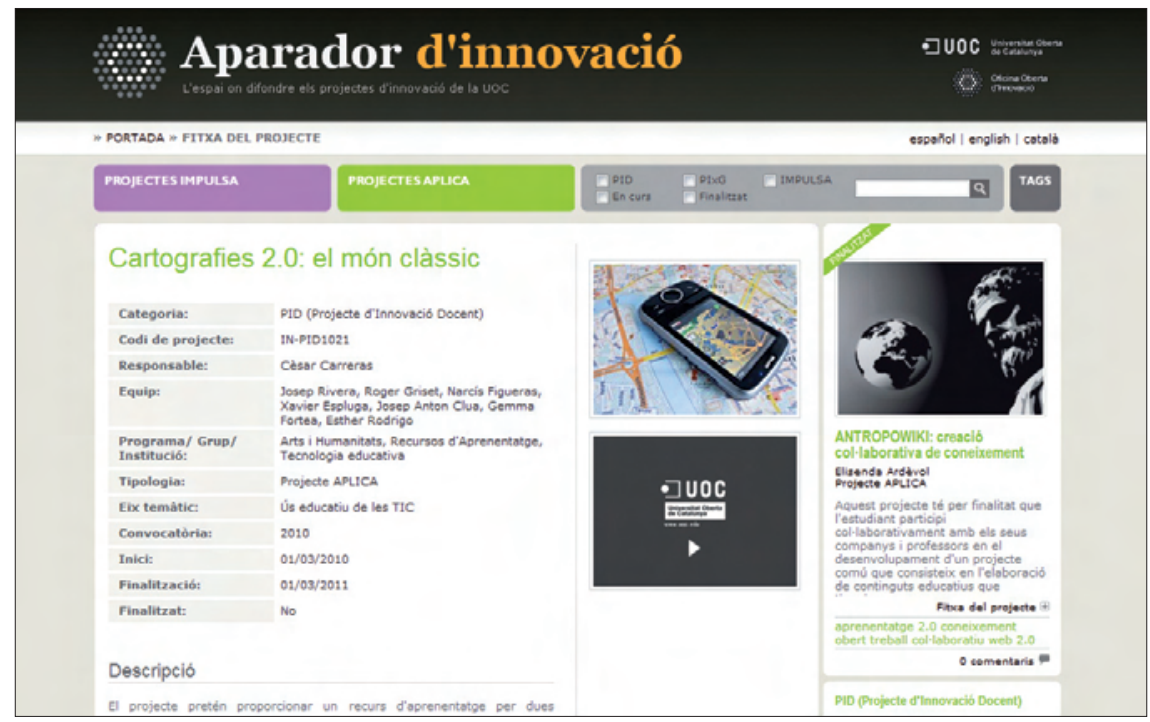

Figura 2. Modelo de ficha desde el portal sectorial público

de cada entorno, permite reducir el coste final (económico y temporal), así como los gastos indirectos resultantes de la administración de varios sitios.

Veamos de forma resumida cuáles son las principales características que permiten agilizar las políticas de creación y publicación de contenidos sin tener que crear costosos desarrollos web paralelos:

- Páginas web basadas en plantillas: utilizando el sistema de administración de contenido se pueden crear, editar y configurar diferentes plantillas de página web para cada necesidad de publicación (ver la ficha de un proyecto en la fig. 1).

Las plantillas del grupo "Publicación web" permiten que los administradores diseñen, distribuyan y gestionen los portales de la intranet de la organización, los sitios web corporativos con presencia en internet, así como los portales departamentales o sectoriales (ver fig. 2). Cada plantilla incluye una barra de herramientas, un editor de textos y funciones de desprotección que pueden ser modificadas a posteriori por el administrador de grupo. Para una edición avanzada de los estilos de cada página existe

predefinidas según las necesidades 
el módulo Office SharePoint Designer 2010, que proporciona un control total sobre las diferentes hojas de estilo aplicadas a cada plantilla o a cada portal.

- Sitio de noticias: para crear una sección de difusión de noticias y poner vínculos de forma rápida y fácil. Permite archivarlas cuando dejan de tener vigencia, un formato de salida estándar para los lectores de noticias RSS, y un módulo llamado "esta semana en imágenes" para generar páginas de fotografías.

- Sitio de publicación con flujo de trabajo a través de un sistema de aprobación. Un validador revisa el contenido antes de ser publicado tanto de forma interna como pública.

- Portal de colaboración: normalmente se utiliza para hospedar sitios de diferentes grupos en un portal de intranet departamental. Desde una página principal se accede a un sitio de noticias, un directorio de sitios y un centro de búsqueda con fichas. Este tipo de plantilla ayuda a crear un portal departamental en el que los empleados pueden colaborar y publicar documentos y páginas web sin necesidad de conocimientos técnicos en edición web, pudiéndose realizar desde un simple documento en Word y su conversión automática a html.

- Portal de publicación: permite crear y gestionar una jerarquía de sitios web o portales de intranet de gran tamaño. Por defecto se parte de una página principal, un subsitio de comunicados de prensa (de ejemplo), un centro de búsqueda y una página de inicio de sesión, todas ellas modificables en función de cada necesidad y cada organización.

\section{Colaboración en la gestión de contenidos}

Aprovechando la capacidad que tiene SharePoint de crear módulos para las diferentes necesidades, se ha desarrollado uno para la gestión de proyectos individuales. Mediante un formulario los miembros de cada equipo de trabajo manejan su documentación de forma sencilla dentro de un área compartida.

Cada área incluye la información básica del proyecto: equipo humano, descripción, objetivos, planificación de tareas mediante un diagrama de Gantt con la definición de las fases, riesgos y planes de contingencia para mitigarlos, y un registro de los problemas presentados donde se anotan las responsabilidades y las soluciones que se han adoptado.

Este módulo ha sido especialmente diseñado para aprovechar las capacidades de trabajo colaborativo que aporta SharePoint y que permiten:

- Compartir documentos, contactos, tareas y calendarios de forma sincronizada con Outlook con miras a realizar el seguimiento de los proyectos.

- Contribuir al intercambio de ideas a través de un wiki, bien sea para colaborar en un diseño en equipo, crear una enciclopedia de conocimientos o, simplemente, recopilar información rutinaria del proyecto de forma sencilla y ágil gracias a que no es necesario tener conocimientos técnicos ni utilizar ningún software específico.

- Compartir entradas en blogs, los cuales son de fácil creación y muy personalizables. Los comentarios a las entra- das pueden activarse (por defecto vienen desactivados), permitiendo a diferentes miembros de una comunidad intercambiar puntos de vista, sugerencias o noticias.

- Hacer el seguimiento de las tareas del proyecto, modificar los hitos y supervisar los posibles desfases desde la visualización compartida del diagrama de Gantt.

- Programar reuniones desde los calendarios, e incluso planificar reuniones de voz y vídeo a distancia gracias a la integración con Office Communicator, que permite varios modos de comunicación simultáneos: chat, videoconferencia, telefonía por VolP, uso compartido de aplicaciones remotas y transferencia de archivos entre los miembros del equipo.

Finalmente, para poder trabajar con un único sistema de gestión de la documentación surgida durante el proyecto, incluye una biblioteca clasificada por carpetas. Los documentos tienen control de versiones, así como protecciones que impiden modificarlos sin autorización. También se ha habilitado la opción de trabajar sin conexión en los archivos de una biblioteca y en los calendarios, contactos, tareas y discusiones. Cuando un usuario vuelve a conectarse todos los archivos del servidor y los elementos de lista se actualizarán automáticamente.

Intranet y cloud computing son conceptos cercanos, puesto que ambos utilizan la misma arquitectura de red basada en internet

\section{Tecnología en la nube, ¿nuevo modelo de intranet?}

Informática en la nube, tecnología en nube o cloud computing (en este trabajo se emplearán indistintamente) es la realización de procesos (almacenamiento, cálculo, tratamiento de datos...) en ordenadores remotos en vez de en la propia institución. Existen empresas que ofrecen este tipo de servicios y sus usuarios se ahorran tener que instalar y mantener equipos informáticos ni programas (para lo cual quizá no son expertos). Los usuarios acceden a través de diferentes dispositivos (ordenadores, móviles, tabletas...).

Intranet y cloud computing son conceptos cercanos, puesto que ambos utilizan la misma arquitectura de red basada en internet, y ponen a disposición de los usuarios la información y servicios de forma remota y deslocalizada. Desde hace unos años se utiliza la tecnología en la nube para crear y/o migrar intranets, sin embargo la cuestión es cómo hacerlo de la mejor forma posible.

Voas y Zhang (2009) escribieron una editorial esclarecedora “Cloud computing: ¿nuevo vino o nueva botella?" en la que invitaban a responder si la informática en la nube era un nuevo paradigma o si era la misma tecnología ya conocida pero en un nuevo envoltorio. Por nuestra parte pensamos que el cloud computing es un nuevo paradigma tecnológico para algo antiguo como son las intranets (Hakan, 2009; Gartner, 2008; Leavitt, 2009). 


\section{Ventajas e inconvenientes del cloud computing}

El coste viene dado por su consumo (pay-as-you-go), por ejemplo, tiempo de uso o funcionalidad, aunque existen servicios gratuitos que se financian con publicidad y donaciones.

Los usuarios no tienen que estar pendientes del mantenimiento ni de las evoluciones y actualizaciones del software y del hardware. La informática en la nube permite rentabilizar las instalaciones, que pueden ser utilizadas las $24 \mathrm{~h}$ del día por usuarios de todo el planeta.

Un posible inconveniente del cloud computing es la programación de las horas de cierre por mantenimiento, que se anuncian pero son ajenas a las necesidades del cliente; sin embargo hoy en día con los sistemas redundados eso es cada vez es menos problemático.

Por otro lado, los argumentos más críticos contra la tecnología en la nube son madurez y seguridad. Se trata de un modelo emergente en plena ebullición, pero debido a que se han producido algunos ataques a instalaciones, la pregunta más incisiva y preocupante a la vez es quién se responsabiliza de los fallos de seguridad o del robo de datos.

Otros puntos conflictivos son:

- el acceso y protección de datos;

- propiedad del conocimiento generado si el servicio deja de funcionar o el cliente se da de baja;

- escasa regulación sobre los derechos de los datos.

La informática en la nube permite rentabilizar las instalaciones de los proveedores y libera a los usuarios de tener que preocuparse de máquinas y programas

\section{El cloud computing desde el punto de vista operativo}

El cloud computing es una de las grandes evoluciones tecnológicas de los últimos años en las TICs. Gartner la considera como la más importante (grand hype) en su lista de las "10 tecnologías estratégicas". Para reforzar esta idea, en el último informe de IDC (Broderick, 2010) se señala al mercado del cloud computing como uno de los más activos, prolijos y con un mayor crecimiento llegando a superar los 2.500 millones de dólares (2,5 billones USA) de facturación en 2015.

Existen 3 tipos de servicios:

\section{- laaS (infrastructure as a service)}

Se trata del hardware como servicio. El proveedor de laaS pone su datacentre al servicio de los clientes para que puedan subir aplicaciones y las ejecuten remotamente en las máquinas del datacentre. Se paga por consumo de recursos (espacio en disco utilizado, tiempo de CPU, transferencia de datos, etc.).

- Paas (platform as a service)

Además de los servicios de infraestructura se incluyen los sistemas operativos, ofreciendo todo lo necesario para soportar el ciclo completo de instalación y puesta en funcionamiento de aplicaciones y servicios web.

- SaaS (software as a service)

Son programas que un proveedor ofrece desde la nube (un gestor de relaciones con los clientes -CRM-, un servicio de correo electrónico...) a los cual acceden los usuarios. Existe una variante que es el S+S (software + service): el SaaS pero además con un cliente instalado localmente que permite trabajar sin conexión (Velte, 2010).

\section{Tipos de nube}

En el modelo de nube pública (ver fig. 3) los recursos informáticos se comparten entre usuarios desconocidos. Permite aprovechar todo el potencial de la nube para soportar grandes proyectos con total flexibilidad y la máxima rapidez a la hora de disponer y gestionar los diferentes recursos, pagando sólo por el uso que de ellos se haga. A nivel de seguridad y disponibilidad -factores clave-, se debe contar con la garantía de que los elementos de red y de hardware están redundados, además de aislados de forma que sólo las personas autorizadas puedan acceder a la información.

Bajo el modelo de nube privada los recursos están disponibles para una sola organización. Se tiene una plataforma escalable en la que se puede decidir el nivel de exclusividad que se requiere, tanto en servidores como en conexiones (Rhoton, 2009). Hay que decidir el grado de aislamiento adecuado, pudiendo optarse por una red privada virtual (VPN) a través de la cual se pueda acceder de forma segura a la infraestructura, garantizando que toda la información que se transfiera viaje de forma cifrada. De esta forma se establece una conexión con nuestros servidores con la misma confiabilidad que en local u on-premise, pero aprovechando las posibilidades de conexión de la nube. A su vez se puede recurrir al aislamiento de los recursos, de forma que toda la infraestructura de hardware (servidores, firewall, balanceadores de carga, sistemas de almacenamiento físico o lógico) esté aislada con dedicación exclusiva, lo que confiere un gran nivel de seguridad e integridad.

Desde el punto de vista de la seguridad cabe destacar que todos los elementos de red (desde el datacentre remoto hasta las comunicaciones, la arquitectura de virtualización, etc.), están redundados para garantizar la máxima disponibilidad del servicio. A este respecto los proveedores deben hacer pública su política y al contratar los servicios se firma un acuerdo de nivel de servicio (service level agreement, $S L A)$, en el que constan las indemnizaciones al cliente en caso de interrupción del servicio. Otro aspecto a tener en cuenta es la ubicación física de los datos pues es un requisito para el cumplimiento de la LOPD (Ley Orgánica de Protección de Datos) en el caso español.

El modelo de nube híbrida por su parte, permite aunar las ventajas de la nube pública y de la nube privada, y contempla la integración del datacentre con otros servidores que no estén en la nube.

\section{Intranets en la nube}

Según un estudio realizado por Forrester Research (Kisker, 2010) en el cuarto trimestre de 2009 , el $24 \%$ de grandes em- 


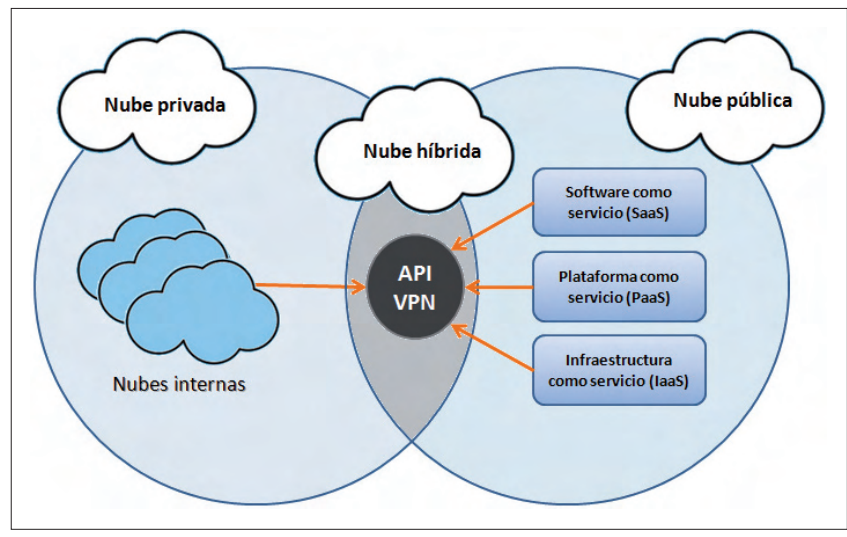

Figura 3. Modelos de cloud computing

presas y pymes de Norteamérica y Europa se manifiestan atraídas por el SaaS o software como servicio como alternativa a los despliegues en sus propias instalaciones, especialmente en aquello referente al concepto de intranet. El ahorro en servidores y costes administrativos, la liberación de los recursos humanos -que se pueden dedicar a tareas más críticas- conforman una serie de valores a favor del cloud computing.

Si bien es cierto que diseñar una estrategia para "subir" servicios clásicos a la nube, como el correo electrónico, no supone ningún problema, en el momento en que nos planteamos esta acción para servicios de mayor envergadura como una intranet, el proceso es mucho más complejo debido a que hemos de contemplar una serie de factores que requieren un análisis exhaustivo y que afectan al diseño de la gestión de documentos, el desarrollo de aplicaciones propias, las secuencias de colaboración o las políticas de publicación interna y externa de contenidos de la intranet corporativa entre otros.

Siendo una intranet una red de ordenadores privados que utiliza la tecnología de internet para compartir sistemas de información y de operaciones, podemos establecer una gran afinidad con el concepto de nube privada tal y como lo hemos definido anteriormente, lo que nos permite centrarnos en la funcionalidad y no en equipos o programas. La intranet desplegada totalmente en la nube da lugar a una clara reducción de costes, pero lo más significativo es el valor que aporta la transformación en la manera de utilizar las TICs en la organización, la escalabilidad y la disponibilidad de servicios bajo demanda (Armbrust, 2010).

Para beneficiarse al máximo de este potencial, el entorno ideal de implementación pasa por el establecimiento de una solución global y completa en la que se pueda ofrecer un sistema de alojamiento y conectividad (laaS), una plataforma de desarrollo (PaaS) -que permita centrarse en las aplicaciones y no en la infraestructura ni en la gestión del software-, y un conjunto de aplicaciones SaaS personalizadas para ejecutarse en el datacentre remoto. Windows Azure Platform integra todos estos elementos, alojados en los datacentres de Microsoft, y pretende no limitar el concepto tecnológico de que el PaaS nació para la nube pública. Ofrece los mismos servicios tanto con el modelo público como privado.

Para ello presenta una plataforma (fig. 4) estructurada en un sistema operativo en la nube como servicio para poder de- sarrollar, administrar y ejecutar las aplicaciones, ofreciendo capacidad de computación, almacenamiento y conectividad (Windows Azure), un sistema de almacenamiento binario (Windows Azure Storage), un gestor relacional (SQL Azure), una solución para conectar servicios cloud y componentes internos a través de aplicaciones (plataforma AppFabric), y un marketplace o repositorio central de información para datos y servicios cloud dentro de la plataforma.

Teniendo en cuenta todas las potencialidades que Windows Azure Platform proporciona, es fácil pensar en abandonar los modelos de implementación actuales en local o en los datacentres públicos. A pesar de que hemos hecho énfasis en más de una ocasión en el efecto de reducción de costes, no debemos pensar únicamente en este punto de vista, y sí en las implicaciones que tiene todo un proceso de migración de servicios, ya que muchos de ellos, con independencia de ser críticos o no, requerirán un nuevo desarrollo específico. Lógicamente, visto el planteamiento de Windows Azure Platform la pregunta que nos podemos plantear es ¿en qué momento podríamos utilizar un modelo como ese? Para dar una respuesta rápida y concisa, y sin menoscabo de otras funciones y aplicaciones posibles, a continuación mencionamos una serie de servicios que pueden residir en un modelo de nube bajo Windows Azure Platform (Li, 2009):

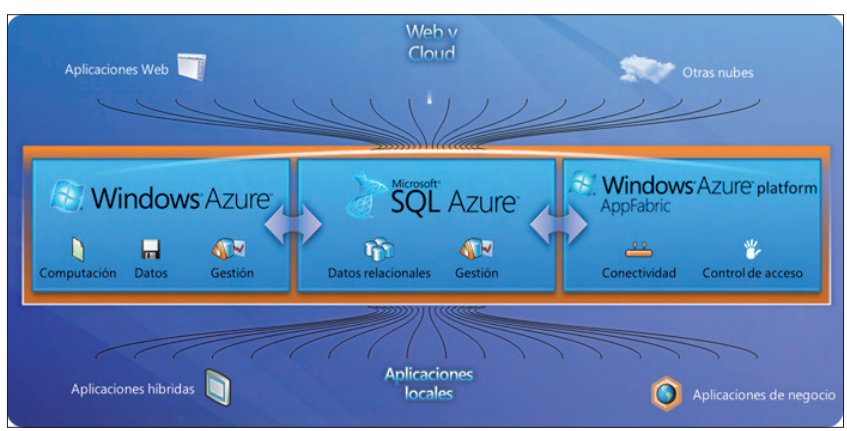

Figura 4. Escenarios de aplicación con Microsoft Windows Azure Platform. Fuente: MSDN Blog, Luis Panzano, http://bit.ly/h6z7J2

\section{Aplicaciones}

- que requieran una escalabilidad dinámica, ajustando en cada momento el servicio a las necesidades reales de la web, como pueda ser la operativa de una aplicación social de alto impacto;

- que requieran una gran fiabilidad y consistencia, tanto de servicios y de datos como de gestión de identidades digitales;

- que tengan una carga variable de computación, donde las puntas de servicio sean controlables;

- con una duración corta o no predecible a priori, que pueda ser puesta en marcha sin afectar a otros procesos;

- de procesamiento paralelo, que generalmente se suelen utilizar de forma puntual;

- sobre las que no se tiene una gran certeza de uso o que necesitan ser escaladas rápidamente ante una demanda masiva;

- que se puedan beneficiar de las bondades del almacenamiento externo, bien sea en forma de copia de seguridad o transaccional no constante. 


\section{Conclusiones}

1. En primer lugar, el concepto tradicional de intranet puede considerarse vigente, por lo menos hasta que internet deje de usar la tecnología que conocemos. Todo lo escrito en los diferentes artículos revisados es totalmente aplicable hoy en día, aspecto que no deja de sorprendernos a tenor de los grandes cambios de hábitos y usos de internet en los últimos diez años.

2. Así mismo, los métodos de diseño e implementación revisados pueden ser utilizados actualmente para construir intranets, en base a la integración de aplicaciones. Siguen siendo válidas las consideraciones universales como acceso transparente al usuario/cliente, gestión de sistemas de recuperación de información, etc., y los métodos de gestión organizacional, arquitectura de información, consultoría de requerimientos, evaluación del uso, etc. Sin embargo en la actualidad existen métodos más novedosos para enfrentarse a la conceptualización y diseño de una intranet.

3. Las intranets se hicieron más complejas al integrar nuevos entornos, funciones y soportes, y tener que tener en cuenta pautas de accesibilidad y usabilidad y una gestión documental más sofisticada.

4. Persiste uno de los eternos problemas: implantar una cultura organizacional basada en la colaboración y la transparencia.

5. El cloud computing permite disponer de más recursos económicos y humanos -al reducirse de forma drástica la inversión en infraestructura-. Una de las grandes ventajas es que un modelo tradicional de intranet en una organización puede ser migrado a la nube para posibilitar un acceso independiente de la ubicación física y de los dispositivos con los que se acceda. No hay que preocuparse por tener una infraestructura o un modelo integrador de aplicaciones internas, sino por las necesidades reales de cómputo de los usuarios. Esa flexibilidad, unida a las tres categorías principales en que se puede dividir el cloud computing, hacen pensar más allá en nuevos planteamientos a la hora de definir una intranet. En la nube se pueden escalar los servicios de almacenamiento e infraestructura de red según necesidades (laaS), disponer de una plataforma para el desarrollo e integración de aplicaciones (PaaS), o crear servicios a medida según las necesidades finales de la organización y de sus usuarios (SaaS).

\section{Nota}

1. La Societat Catalana de Documentació i Informació (Socadi) creada en 1984 a imagen de Sedic, funcionó hasta 1999, en que se fusionó al Cobdc, contando entonces con 350 asociados.

\section{Bibliografía}

Abadal, Ernest. "Intranets documentales". El profesional de la información, 1998, junio, v. 7, n. 6, pp. 35-38.

http://www.elprofesionaldelainformacion.com/contenidos/ 1998/junio/intranets_documentales.html

Alòs-Moner, Adela. "Intranets: sonrisas y lágrimas". El profesional de la información, 2002, enero-febrero, v. 11, n. 1, pp. 4-8. http://www.elprofesionaldelainformacion.com/contenidos/ 2002/enero/1.pdf

Armbrust, Michael; Fox, Armando; Griffith, Rean; et al. "A view of cloud computing". Communications of the ACM, 2010, April, v. 53, n. 4, pp. 50-58.

DOI: $10.1145 / 1721654.1721672$

Broderick, Katherine; Bailey, Michelle; Eastwood, Matthew. Worldwide enterprise server cloud computing 2010 2014 forecast. IDC, April 2010.

Bustelo-Ruesta, Carlota; García-Morales-Huidobro, Elisa; García-de-Paso-Gómez, Esperanza. "Definición del modelo estratégico de desarrollo de una intranet corporativa: metodología basada en un caso práctico". El profesional de la información, 2006, septiembre-octubre, v. 15, n. 5, p. 352362.

http://www.elprofesionaldelainformacion.com/contenidos/ 2006/septiembre/4.pdf

Gartner, Inc. "Cloud computing will be as influential as ebusiness", 2008.

http://www.gartner.com/it/page.jsp?id=707508

Hakan, Erdogmus. "Cloud computing: Does nirvana hide behind the nebula?" IEEE software, 2009, v. 26, n. 2, pp. 4-6. DOI: 10.1109/MS.2009.31

IDC. Cloud computing market will reach \$2.5bn by 2015 http://www.womenintechnology.co.uk/news/idc-cloudcomputing-market-will-reach-2-5bn-by-2015-news800385453

Kisker, Holger; Ried, Stefan; Shey, Heidi. The state of enterprise software and emerging trends: 2010. Forrester Research, 2010.

Leavitt, Neal. "Is cloud computing really ready for prime time?" Computer, 2009, v. 42, n. 1, pp. 15-20.

DOI: 10.1109/MC.2009.20

Li, Henry. Introduction to Windows Azure: an introduction to cloud computing using Microsoft Windows Azure. Berkeley: Apress; New York: Springer-Verlag New York, cop. 2009.

DOI: 10.1007/978-1-4302-2470-9_4

Nielsen, Jakob. 10 best intranets of 2011. 2011. http://www.useit.com/alertbox/intranet_design.html

Pérez-González, Daniel; Solana-González, Pedro. “Intranets medición y valoración de sus beneficios en las organizaciones". El profesional de la información, 2006, septiembre-octubre, v. 15, n. 5, pp. 331-341.

http://www.elprofesionaldelainformacion.com/contenidos/ 2006/septiembre/2.pdf

Rhoton, John. Cloud computing explained: handbook for enterprise implementation. London: Recursive Ltd., 2009.

Velte, Anthony; Velte, Toby J.; Elsenpeter, Robert C. Cloud computing: a practical approach. New York: McGraw-Hill, 2010.

Voas, Jeffrey; Zhang, Jia. "Cloud computing: new wine or just a new bottle?", IT professional, 2009, Mar./Apr., v. 11, n. 2, pp. 15-17.

DOI: 10.1109/MITP.2009.23 


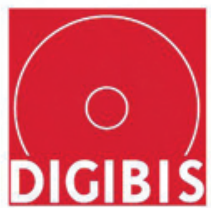

\section{Le ayudamos a crear Bibliotecas Virtuales}

desde la digitalización de materiales bibliográficos hasta la asignación de metadatos y su implementación en la red, conforme a la normativa internacional.

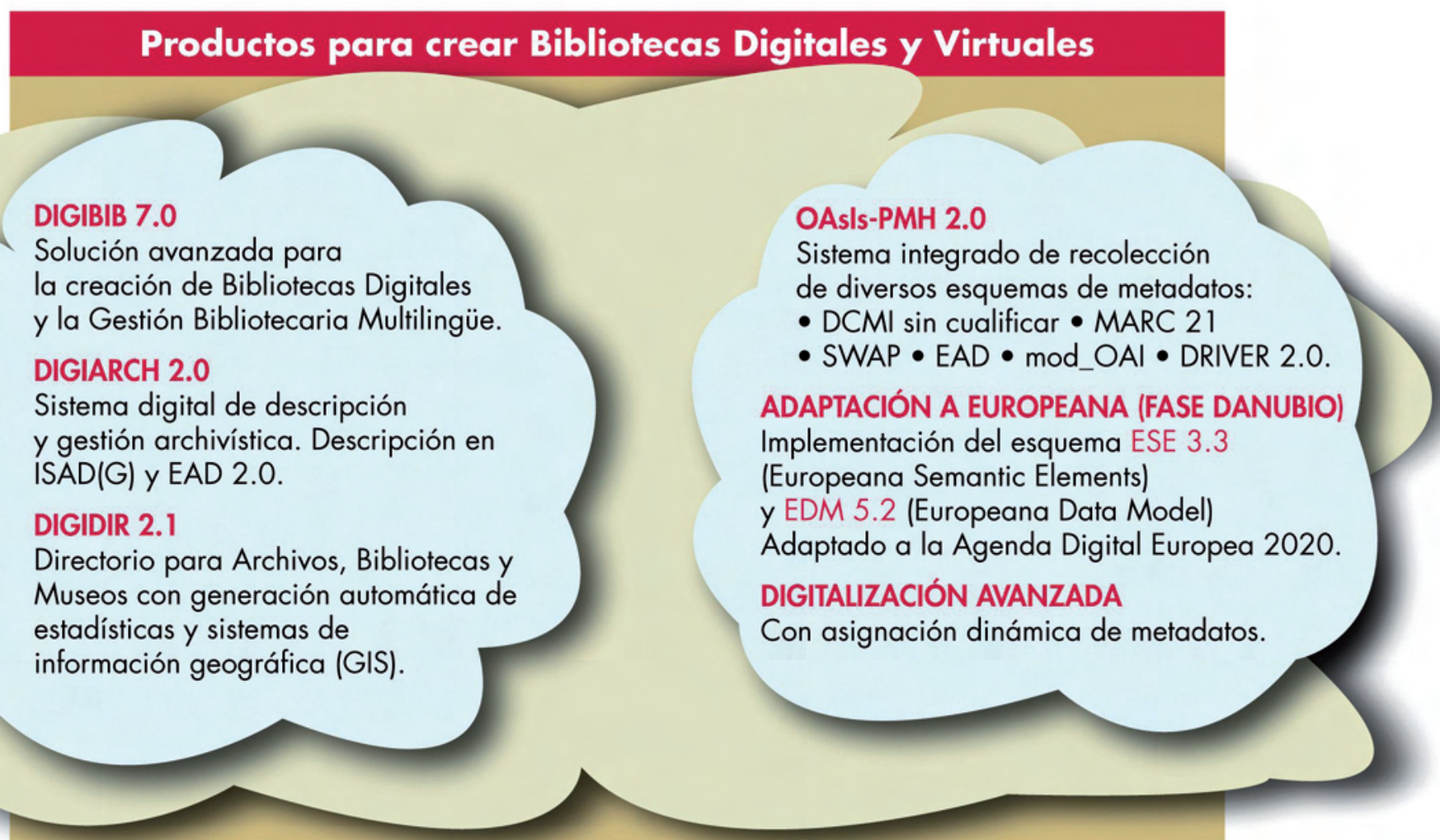

- Recolección en la Web para Entidades e Instituciones de Memoria en OAI-PMH y Dublin Core cualificado con ESE 3.3

Consultoría y mappings a EDM 5.2 (Europeana Data Model)

Tecnologías abiertas para la creación, recuperación y recolección de metadatos (MARCXML, DCMI y RDF y RDFs)

Servidor adicional de SRU/OpenSearch para Europeana

Adaptación del repositiro OAl para la transmisión de instancias RDF según ORE

Repositorios Institucionales DIGIPRESV para Preservación Digital a largo plazo mediante PREMIS 2.0 y OAIS ISO 14721

WBC

- Intercambio de metadatos en METS 1.9 (diferentes Profiles) integrando todos los esquemas de metadatos

- Creación de METSRights para el control de los derechos de autor

SKOS

- Reconocimiento Óptico de Caracteres OCR y generación dinámica de ALTO (Analyzed Layout and Text Object)

- Generación e integración de registros SKOS mediante MARC21(Up. 12)/RDA

- Creación de eBooks o libros digitales en formatos: ePub y Mobipocket.

Adaptación de DIGIBIB a linked Open Data 